\title{
Relación entre el Patrón Venoso Superficial del Antebrazo y las Complicaciones de los Cateterismos Venosos Periféricos y Centrales en una Muestra de Niños Atendidos en una Unidad de Cuidados Intensivos Pediátricos de Santander Colombia
}

\author{
Relationship between Surface Forearm Vein Pattern and Complications of Peripheral and Central Venous \\ Catheterization in a Sample of Children Treated in the Pediatric Intensive Care Unit of Santander Colombia
}

Edgar Giovanni Corzo Gómez*; Martha Isabel Robles Carreño**; Ángela Milena Díaz Díaz ${ }^{* * *}$ \& Sonia Esperanza Osma Zambrano*****

CORZO, G. E. G.; ROBLES, C. M. I.; DÍAZ, D. A. M. \& OSMA, Z. S. E. Relación entre el patrón venoso superficial del antebrazo y las complicaciones de los cateterismos venosos periféricos y centrales en una muestra de niños atendidos en una unidad de cuidados intensivos pediátricos de Santander, Colombia. Int. J. Morphol., 34(3):885-889, 2016.

RESUMEN: Desde hace varias décadas se han adelantado estudios anatómicos que muestran como las poblaciones en diversos países mantienen una representación de los diferentes patrones venosos superficiales en el antebrazo. Esto contrasta con el concepto arraigado en los textos clásicos de anatomía que mantuvieron la idea de una poca variabilidad de las venas y un predominio del llamado patrón en "M". El presente estudio descriptivo, en niños que ingresaron a la Unidad de Cuidados Intensivos Pediatricos (UCIP) y requirieron acceso venoso en miembro superior, busca determinar si existe asociación entre las venas y el patrón venoso superficial del antebrazo y la necesidad de retirar los catéteres superficiales (bránula y epicutáneo). Se incluyeron 54 pacientes: 23 niñas y 31 niños. Se valoraron las indicaciones de venopunción y el tipo de patrón venoso. Se presentaron 22 cambios en la venopunción principalmente en los patrones II y IV. Pero aunque el patrón IV tuvo un porcentaje de presentación menor comparado con el patrón II, fue el único patrón en el cual se presentó la necesidad de cambiar la venopunción en 3 ocasiones consecutivas. Consideramos de gran importancia darle una aplicación clínica al concepto anatómico de los patrones venosos; fomentar en las escuelas de Enfermería y Medicina la enseñanza de los patrones venosos y sus implicaciones de riesgo, y valorar con estudios posteriores si el patrón tipo IV puede aumentar el riesgo de iatrogenia en poblaciones infantiles.

PALABRAS CLAVE: Patrón venoso; Venas superficiales; Vena cefálica; Vena Basílica; Cateterismo venoso.

\section{INTRODUCCIÓN}

Los pacientes de la Unidad de Cuidados Intensivos Pediátricos (UCIP) y/o neonatales requieren con frecuencia de una vía venosa confiable y segura. El procedimiento más utilizado para obtener esta vía es la venopunción periférica, sin embargo, en ocasiones se hace necesario la instalación de una vía venosa central denominada Catéter Venoso Epicutáneo (CVE). El CVE es un catéter venoso central de acceso periférico cuya técnica es realizada por el personal de Enfermería. Según Giraldo-Montoya et al. (2008) este procedimiento garantiza una vía venosa más duradera y confiable por la cual se puede administrar líquidos, medica- mentos y nutrientes. La terapia endovenosa puede conllevar complicaciones que pueden presentarse durante la instalación del catéter, en la administración de la terapia o al retirar el catéter. Como lo señala Cartwright (2004) estas complicaciones incluyen flebitis química, flebitis mecánica, flebitis bacteriana, infiltración o extravasación, hematoma, tromboembolia y punción accidental de una arteria o un nervio. Se ha sugerido que la morfología y organización de las venas superficiales del antebrazo puede incidir en la frecuencia y número de las complicaciones, bien sea en el momento de la inserción del catéter o durante el tiempo de

\footnotetext{
Médico, Mcs. Ciencias Básicas Biomédicas. Docente de Morfología, Universidad Autónoma de Bucaramanga, Bucaramanga, Colombia.

** Enfermera Jefe, Docente Enfermería, Universidad Autónoma de Bucaramanga, Bucaramanga, Colombia.

*** Coordinadora Médica UCIP y Pediatría Clínica FOSCAL, Docente Programa de Medicina, Universidad Autónoma de Bucaramanga, Bucaramanga, Colombia.

***** Médico, Ginecóloga, Mcs. en Epidemiología. Docente Medicina, Universidad Autónoma de Bucaramanga, Bucaramanga, Colombia. Proyecto 134021 de convocatoria interna de la universidad Autónoma de Bucaramanga (UNAB).
} 
tratamiento parenteral. Las complicaciones de las venopunciones generan cambio en el sitio de punción lo cual aumenta el riesgo de infecciones y de dolor en los niños Racadio et al. (2001). En términos de seguridad para el paciente es importante determinar todas las posibles causas que generen aumento en las complicaciones de las venopunciones y los elementos anatómicos no deben ser una excepción. Los estudios morfológicos de del Sol et al. $(1988,1990,2007)$ aunado a otros autores latinoamericanos han permitido describir cinco patrones anatómicos de distribución de las venas superficiales en el miembro superior; este conocimiento se ha ido incorporando en la nuevas generaciones de enfermeros y auxiliares de enfermería buscando que su aplicación disminuya el número de eventos iatrogénicos.

El presente estudio busca determinar si existe asociación entre las venas y el patrón venoso superficial del antebrazo y la necesidad de retirar los catéteres superficiales (bránula y epicutáneo) en los niños atendidos en la unidad de cuidados intensivos pediátricos (UCIP) de la Clínica FOSCAL.

Proyecto 134021 de convocatoria interna de la universidad Autónoma de Bucaramanga (UNAB).

\section{MATERIAL Y MÉTODO}

Estudio descriptivo de cohorte prospectiva, exploratorio, en niños que ingresaron a la UCIP y requirieron acceso venoso en miembro superior entre Marzo a Octubre del 2014. Se seleccionaron los pacientes por conveniencia y se describió el patrón venoso, la vena puncionada y la indicación de la venopunción. Posteriormente se registraron las causas que hicieron necesario retirar el catéter para realizar una nueva venopunción. Para valorar las venas puncionadas se usó un visualizador de venas Accuvein el cual fue facilitado por la empresa Becton Dickinson, Medical - Medication \& Procedural Solutions. Se excluyeron los accesos diferentes como: catéter umbilical, arterial, o sitio diferente al miembro superior.

Teniendo en cuenta la clasificación de patrones venosos superficiales propuesta por el del Sol et al. (2007), se determinó el sitio de punción en mano o antebrazo, número de cambios y el motivo de dicho cambio de la venopunción (Fig. 1).

Se estimó la frecuencia de las variables cuantitativas mediante promedios y desviación estándar si la distribución

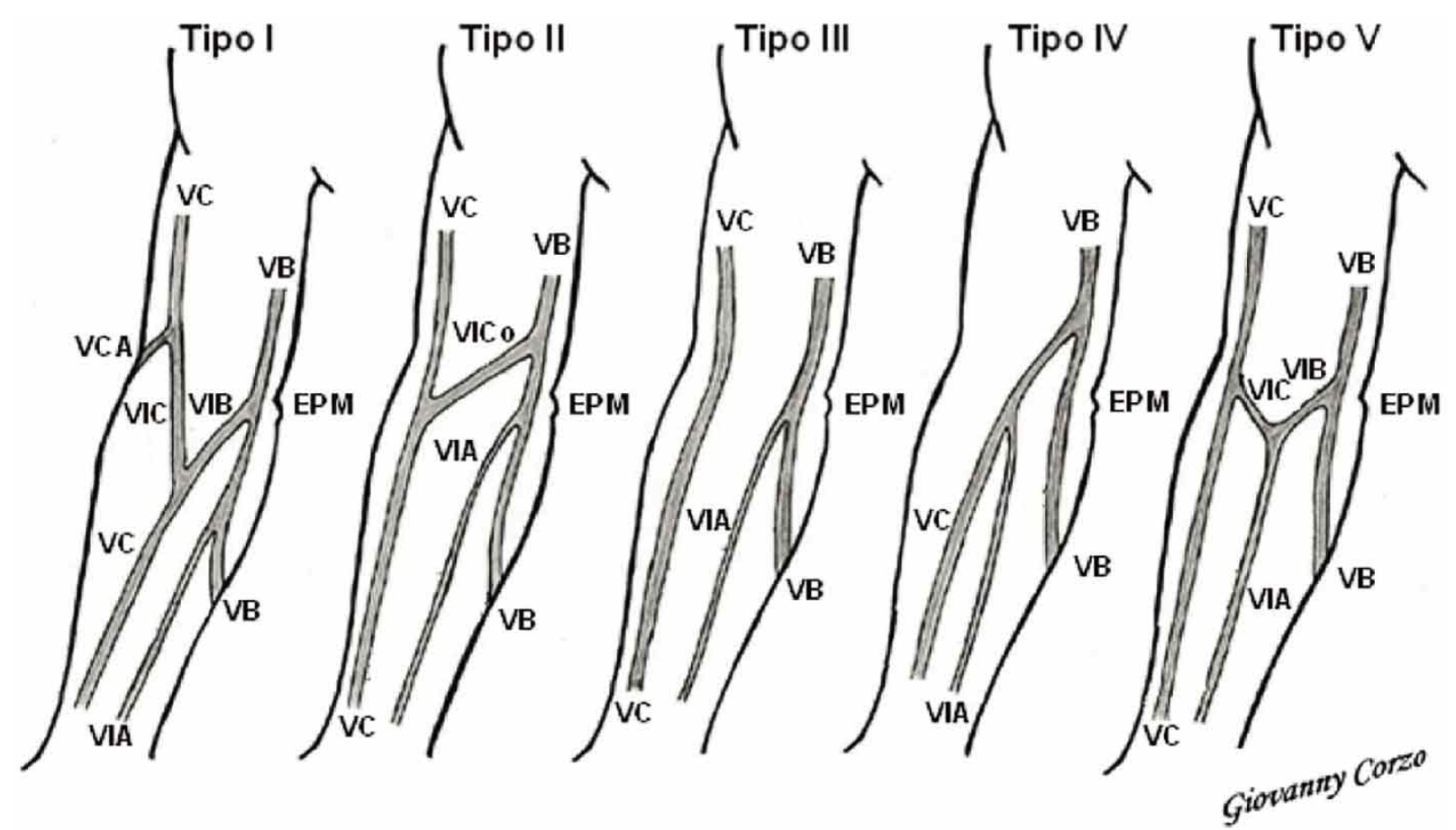

Fig. 1. Descripción de los patrones venosos de la fosa cubital según la propuesta de del Sol et al. $(1988,2007)$. VC= vena cefálica; VB= vena basílica; VCA = vena cefálica accesoria; VIC = vena intermedia cefálica; VIB= vena intermedia basíli$\mathrm{ca} ; \mathrm{VIA}=$ vena intermedia del antebrazo; $\mathrm{VICo}=$ vena intermedia del codo; $\mathrm{EPM}=$ epicóndilo medial. 
CORZO, G. E. G.; ROBLES, C. M. I.; DÍAZ, D. A. M. \& OSMA, Z. S. E. Relación entre el patrón venoso superficial del antebrazo y las complicaciones de los cateterismos venosos periféricos y centrales en una muestra de niños atendidos en una unidad de cuidados intensivos pediátricos de Santander, Colombia. Int. J. Morphol., 34(3):885-889, 2016.

es paramétrica y mediana con el rango interquartil en caso de variables no paramétricas. Se estimó la prevalencia de los diferentes patrones venosos. En el análisis bivariado se estableció asociación estadística entre presencia de una complicación y/o presencia de una complicación específica y el tipo de patrón, la presencia de patrón IV, la vena puncionada, la indicación del tratamiento, número de cambios de la veno-punción y demás variables mediante la prueba de chi cuadrado.

\section{RESULTADOS}

Se valoraron 54 pacientes: 23 niñas y 31 niños, $75 \%$ de los pacientes tenían un peso menor de 3500 g. El $87 \%$ (47) de las venopunciones fueron periféricas (bránula) y 13 \% (7) catéter epi-cutáneos. Las indicaciones para la venopunción incluyeron hidratación, flujo metabólico, administración de bolos, antibioticoterapia, analgesia o sedación, transfusión de hemoderivados, administración de inotrópicos y nutrición. En relación al tipo de patrón encontrado $28 \%$ (15) fueron tipo I o tipo II, $16 \%$ (9) tipo III o tipo IV y $12 \%$ (6) de tipo V (Tabla I).

Se presentaron veintidós cambios de la venopunción por diferentes motivos como: salida de bránula, presencia de edema, flebitis mecánica, flebitis química y obstrucción; $27 \%$ de los pacientes (15) requirieron un cambio, $11 \%$ (6) dos cambios y $1,8 \%$ (1) tres cambios. En las 15 venopunciones que requirieron un cambio los patrones que

Tabla I. Frecuencia de patrones venosos superficiales en la cohorte estudiada.

\begin{tabular}{lcc}
\hline Patrón venoso & Frecuencia & Porcentaje (\%) \\
\hline I & 15 & 28 \\
II & 15 & 28 \\
III & 9 & 16 \\
IV & 9 & 16 \\
V & 6 & 12 \\
Total & 54 & 100 \\
\hline
\end{tabular}

predominaron fueron el II y el IV. En los 6 pacientes que requirieron dos cambios predominaron los patrones I y II. El paciente que requirió tres cambios presentó patrón IV. Del total de cambios de venopunción, el $27 \%$ correspondían a patrón tipo II y el 27 \% a patrón tipo IV (Tabla II).

No se encontró asociación entre presencia de complicación y el resto de covariables, pero si una asociación significativa entre la presencia de patrón venoso tipo IV y la presencia de flebitis $(\mathrm{p}<0,05)$ como causa para cambiar la venopunción.

\section{DISCUSIÓN}

Los patrones II y IV requirieron el mayor número de cambios de la venopunción, de esto llama la atención la alta frecuencia de cambios requeridos en el patrón tipo IV en razón a que sólo estaba presente en el $16 \%$ (9) del total de pacientes en tanto que el patrón tipo II estaba presente en el $28 \%$ (15) del total. De hecho el comportamiento de las frecuencias de presentación de los patrones venosos guarda relación con la mayoría de los reportes hechos a nivel de Latinoamérica en los cuales predominan los patrones I, II y III en su respectivo orden mientras el patrón IV es de menor frecuencia, y patrón V (en "M") es el de menor presentación (del Sol et al., 1988, 1990, 2007, 2012). De igual manera se relaciona con los hallazgos previos en población colombiana del Departamento de Santander y de su capital Bucaramanga (Corzo Gómez et al., 2010, 2014).

Sin embargo la frecuencia del patrón V $12 \%$ (6) es un poco alta comparativamente con los hallazgos previos los cuales se han reportado con valores que oscilan entre el $3 \%$ y el $8 \%$. En un estudio realizado con tomografía computarizada helicoidal en individuos chilenos se encontró una frecuencia similar de 11,7 \% (del Sol et al., 2012).

Los sitios más comúnmente usados para los accesos venosos periféricos en el miembro superior se ubican en la región distal (Loro Sancho et al., 2005) señalan que se debe

Tabla II. Número de veces que se requirió cambio de la venopunción de acuerdo al patrón venoso superficial del antebrazo.

\begin{tabular}{lcccc}
\hline Patrón venoso & \multicolumn{2}{c}{ Número de veces que requirió cambio de la venopunción } & $\begin{array}{c}\text { Total de cambios } \\
\text { de la venopunción }\end{array}$ \\
\cline { 2 - 4 } & Un cambio & Dos cambios & Tres cambio & $5(23 \%)$ \\
Patrón I & $3(20 \%)$ & $2(33 \%)$ & $0(0 \%)$ & $6(27 \%)$ \\
Patrón II & $4(27 \%)$ & $2(33 \%)$ & $0(0 \%)$ & $3(14 \%)$ \\
Patrón III & $2(13 \%)$ & $1(17 \%)$ & $0(0 \%)$ & $6(27 \%)$ \\
Patrón IV & $4(27 \%)$ & $1(17 \%)$ & $1(100 \%)$ & $2(9 \%)$ \\
Patrón V & $2(13 \%)$ & $0(0 \%)$ & $0(0 \%)$ & $22(100 \%)$ \\
Total & $15(100 \%)$ & $6(100 \%)$ & $1(100 \%)$ & \\
\hline
\end{tabular}


CORZO, G. E. G.; ROBLES, C. M. I.; DÍAZ, D.A. M. \& OSMA, Z. S. E. Relación entre el patrón venoso superficial del antebrazo y las complicaciones de los cateterismos venosos periféricos y centrales en una muestra de niños atendidos en una unidad de cuidados intensivos pediátricos de Santander, Colombia. Int. J. Morphol., 34(3):885-889, 2016.

iniciar con las venas del dorso de la mano, continuar con el antebrazo y luego con las venas de la flexura del codo en razón a que si se oblitera una vena canalizada no se inhabilitan las otras venas más distales. Algunos estudios morfológicos han puesto énfasis en la importancia de valorar la vena a puncionar de acuerdo al patrón venoso superficial del antebrazo de cada paciente.

Para Barria \& Santander (2006) quienes valoraron las complicaciones en 828 dispositivos de acceso vascular periférico en una unidad de cuidado intensivo, el 36,5\% fueron instaladas en las venas del arco venoso dorsal de la mano y el 19,2 \% en las venas de la fosa cubital. Cárcoba Rubio \& Ceña Santorcuato (2010) recomiendan que para instalar un catéter venoso central de acceso periférico en la fosa antecubital, elegir preferentemente el miembro superior derecho y las venas basílica, mediana (intermedia del antebrazo) y cefálica, en este orden, puesto que la vena basílica del brazo derecho es la de acceso más directo anatómicamente al atrio derecho, y por tanto, la introducción del catéter sería más sencilla.

del Sol \& Olave (1991) luego de estudiar 400 antebrazos de jóvenes chilenos recomendaron que para la punción venosa de la fosa cubital se usara preferiblemente la vena intermedia basílica (VIB) o la vena intermedia del codo (VICo) teniendo en cuenta su estrecha relación con el nervio cutáneo medial del antebrazo al igual que con la arteria braquial y el nervio mediano. Por otra parte si el calibre de la vena cefálica (VC) o de la vena intermedia cefálica (VIC) es semejante al de la VIB o VICo, es recomendable la utilización de ellas ya que no tienen relaciones topográficas de importancia con elementos vasculares y nerviosos más que con el nervio cutáneo lateral del antebrazo.

Queremos destacar que dentro de las recomendaciones que se hacen en las guías de Enfermería y manuales de procedimiento de las Instituciones de Salud se enfatiza en medidas como las mencionadas por Ochoa et al. (2004), las cuales incluyen localizar y seleccionar la vena a canalizar de acuerdo al propósito de la terapia intravenosa, palpándola con la yema de los dedos y evitar la utilización de aquellas que tengan las siguientes características: punción previa reciente, anormalidades anatómicas, rigidez, demasiada movilidad, inflamación o tromboflebitis.

Luego de los estudios adelantados en varios países latinoamericanos hay claridad sobre el hecho de que en todas las poblaciones hay presencia en mayor o menor frecuencia de estos patrones venosos. También del hecho que el patrón venoso denominado en " $\mathrm{M}$ " no tiene una representación tan grande como se pensaba hace varias décadas. Infortunadamente los textos anatómicos han obviado la im- portancia de los patrones venosos superficiales y esto ha llevado a que el personal de enfermería y auxiliares de enfermería tengan un aprendizaje sesgado de la anatomía venosa. El abordaje de una venopunción sin el conocimiento completo de la anatomía puede generar lesiones sobre estructuras vasculares y nerviosas adyacentes a las venas tal como lo señalaron Yamada et al. (2008).

Consideramos de gran importancia que el personal de Enfermería se familiarice con la morfología de los patrones venosos, sus características, los riesgos que representa cada uno de ellos y que en particular, se le diera importancia al que denominamos en este artículo como "patrón tipo IV" en razón a que, la "curva" que hace la vena cefálica para unirse a la vena basílica puede predisponer a extravasación del catéter periférico o central en el momento de la instalación. Lo anterior es de mayor importancia en los niños atendidos en unidades de cuidados intensivos ya que son de menor talla y en ellos, el movimiento de la punta del catéter sólo unos milímetros representa más riesgo debido a la corta longitud de sus antebrazos.

\section{AGRADECIMIENTOS}

Agradecemos a la Universidad Autónoma de Bucaramanga por su apoyo y financiación al proyecto. También al personal médico y de Enfermería de la Unidad de Cuidados intensivos pediátricos de la Clínica FOSCAL en donde se realizó el proyecto. De igual manera a la empresa Becton Dickinson, Medical - Medication \& Procedural Solutions la cual prestó el visualizador venoso que permitió valorar los patrones venosos de los bebés sin tocarlos.

CORZO, G. E. G.; ROBLES, C. M. I.; DÍAZ, D. A. M. \& OSMA, Z. S. E. Relationship between surface forearm vein pattern and complications of peripheral and central venous catheterization in a sample of children treated in the pediatric intensive care unit of Santander, Colombia. Int. J. Morphol., 34(3):885-889, 2016.

SUMMARY: For several decades there have been advanced anatomical studies showing how populations in different countries maintain a representation of the different surface vein patterns in the forearm. This contrasts with the concept rooted in the classical texts of anatomy that kept the idea of a low variability of veins and a predominance of pattern called "M". This descriptive study in children admitted to the Pediatric Intensive Care Unit (PICU) and required upper limb venous access, seeks to determine whether there is an association between the veins and superficial venous pattern of the forearm and the need to remove the catheter surface (branule and epicutaneous). Fifty-four patients were included: 23 girls and 31 boys. Directed by venipuncture and type 
of vein patterns were assessed. Twenty-two changes occurred mainly in venipuncture patterns II and IV. But although the pattern IV had a lower percentage compared to the standard presentation II, it was the only pattern which showed the need to change the venipuncture on 3 consecutive occasions. We assign great importance to give a clinical application to the anatomical concept of vein patterns; encouraging nursing schools and those teaching Medicine vein patterns and associated risk implications, and titrate with further studies if the IV pattern can increase iatrogenic risks in pediatric populations.

KEY WORDS: Vein pattern; Superficial veins; Cephalic vein; Basilic vein; Venous catheterization.

\section{REFERENCIAS BIBLIOGRÁFICAS}

Barria P., M. R. \& Santander M., G. M. Acceso vascular periférico en neonatos de cuidado intensivo: Experiencia de un hospital público. Cienc. Enferm., 12(2):35-44, 2006.

Cárcoba Rubio, N. \& Ceña Santorcuato, S. Cateterización venosa central de acceso periférico mediante técnica Seldinger modificada en la urgencia hospitalaria. Enferm. Glob., (20):1- 8, 2010 .

Cartwright, D. W. Línea venosa central en recién nacidos: un estudio de 2186 catéteres. Arch. Dis. Child. Fetal Neonatal Ed., 89(6):f504-8, 2004

Corzo Gómez, E. G; Gómez Díaz, O. L.; Niño Mantilla, M. E.; Rey Triana, R. J. \& Pedraza Díaz, L. J. Distribution pattern of the veins of the cubital fossa in a sample of people born in Bucaramanga, Colombia. Int. J. Morphol., 28(4):1011-8, 2010.

Corzo Gómez, E. G; Gómez Díaz, O. L.; Niño Mantilla, M. E.; Ramírez Vargas, L. M. \& Zárate Sierra, L. M. Distribution pattern of the veins of the cubital fossa in a sample of people born in the department of Santander, Colombia. Int. J. Morphol., 32(1):221-6, 2014.

del Sol, M.; DeAngelis, M. A. \& Bolini, P. A. D. Formações venosas na fossa cubital da criança. Pediatr. Mod., 23(4):225-31, 1988.

del Sol, M.; Wuster, A. \& Fritz, I. Tipos de formaciones venosas en la fosa cubital en jóvenes chilenos. Rev. Chil. Tecnol. Méd., 13(2):646-9, 1990.

del Sol, M. \& Olave, E. Venas de la fosa cubital en el hombre. Sitios de punción. Rev. Chil. Cienc. Med. Biol., 1(1):49-53, 1991.

del Sol, M.; Lagos Mardones, M. \& Torres Bustos, E. Venous formations in the cubital fossa of mapuche. Bioscopy study. Int. J. Morphol, 25(4):885-94, 2007. del Sol, M.; Lillo, E.; Lobos, L. \& Vásquez, B. Study of the veins of the cubital fossa by helical computed tomography and its clinical application. Int. J. Morphol., 30(1):64-9, 2012.

Giraldo-Montoya, D. I.; Quirós-Jaramillo, A. \& Mejía-Cadavid, L. A. Manejo de catéteres centrales de inserción periférica en recién nacidos. Aquichán, 8(2):257-65, 2008.

Loro Sancho, N.; Sancho Sánchez, M. J.; Sancho Sánchez, M. T.: Martínez Hernández, E. \& Peiró Andrés, A. Enfermería: Canalizaciones periféricas, atención, cuidados, mantenimiento y complicaciones. Enferm. Glob., (7):1-19, 2005.

Ochoa, R. V.; Arroyo de Cordero, G.; Lee G., M.; Jiménez Sánchez, J.; Galindo Barrera, M.; Hernández García, G.; Sánchez González, J. M.; Hernández Gamboa, L. E.; Campos Castolo, E. M.; Chávez Villanueva, M.; Garnica Fonseca, R.; Barragán Sosa, P. I.; Ortíz Ensástegui, M. T.; Olguín Tavera, P.; Suárez Vázquez, M.; Jasso Ortega, G.; Ibarra Castañeda, G. \& Tena Tamayo, C. Recomendaciones específicas para enfermería sobre el proceso de terapia endovenosa. Rev. Mex. Enferm Cardiol., 13(1-2):53-60, 2005.

Racadio, J. M.; Doellman, D. A.; Johnson, N. D.; Bean, J. A. \& Jacobs, B. R. Pediatric peripherally inserted central catheters: complication rates related to catheter tip location. Pediatrics, 107(2):E28, 2001.

Yamada, K.; Yamada, K.; Katsuda, I. \& Hida, T. Cubital fossa venipuncture sites based on anatomical variations and relationships of cutaneous veins and nerves. Clin. Anat., 21(4):307-13, 2008.

\author{
Dirección para Correspondencia: \\ Edgar Giovanni Corzo Gómez \\ Universidad Autónoma de Bucaramanga \\ Calle 157 número 19-55 \\ Cañaveral Parque Floridablanca \\ Santander \\ COLOMBIA
}

Email: ecorzo@unab.edu.co

Recibido : 01-07-2016

Aceptado: 04-05-2016 